Bulletin of the Section of Logic

Volume 47/1 (2018), pp. 45-58

http://dx.doi.org/10.18778/0138-0680.47.1.04

Zalán Gyenis

\title{
ALGEBRAIC CHARACTERIZATION OF THE LOCAL CRAIG INTERPOLATION PROPERTY
}

\begin{abstract}
The sole purpose of this paper is to give an algebraic characterization, in terms of a superamalgamation property, of a local version of Craig interpolation theorem that has been introduced and studied in earlier papers. We continue ongoing research in abstract algebraic logic and use the framework developed by AndrékaNémeti and Sain.
\end{abstract}

Keywords: Craig interpolation, Algebraic logic, Superamalgamation 2010 Mathematics Subject Classification: 03G27

\section{Introduction}

The aim of this paper is to give an algebraic characterization of a local version of Craig's interpolation theorem that has been earlier studied in [12], [17]. We use the framework of universal algebraic logic as introduced in $[1,2]$. This approach is strongly related to that of Blok and Pigozzi [5, 4, 6, 19], Czelakowski [9], Font-Jansana [10, 11], and Henkin-MonkTarski [13]. However, to keep the present work self-contained we briefly recall the indispensable definitions and theorems in Section 2. In algebraic logic one is interested in (i) finding a general method for assigning a class of algebras $\operatorname{Alg}(\mathbf{L})$ to a logic $\mathbf{L}$, and (ii) having such a method one wishes to establish equivalence theorems of the following kind

Logic $\mathbf{L}$ has property $P \quad \Longleftrightarrow \quad \operatorname{Alg}(\mathbf{L})$ has property $\operatorname{Alg}(P)$.

For example, if $\mathbf{L}$ is a modal $\operatorname{logic}$, then $\operatorname{Alg}(\mathbf{L})$ is a class of Boolean algebras with operators, where the extra Boolean operators correspond to 
the modalities of $\mathbf{L}$. Many such equivalence results have been established in the literature so far. Very rough examples are: completeness theorems for logics correspond to representation theorems for algebras; compactness properties correspond to the class of algebras being closed under ultraproducts; Beth definability theorems correspond to certain epimorphisms between algebras being surjective. For details we suggest $[1,2]$ and references therein: the literature contains similar theorems for a very large number of further logical properties.

Craig interpolation property has been investigated ever since Craig proved that this property holds for usual first order logic. Related problems have been intensively studied in the literature of algebraic logic. It turned out that interpolation properties of different logics are strongly related to various amalgamation properties of certain classes of algebras. We refer to Pigozzi [18], Sain [20] and to Sági-Shelah [21] and references therein. Craig interpolation property has two major versions: $(\models$ Craig) and $(\rightsquigarrow$ Craig).

A logic $\mathbf{L}^{1}$ is said to have $(\models$ Craig) property (see [14]) if whenever $\phi \models$ $\psi$ there is $\chi$ using only propositional letters common in $\phi$ and $\psi$ such that $\phi \models \chi$ and $\chi \models \psi$. It has been shown in Czelakowski [7] (Theorem 3) that $(\models$ Craig) interpolation property of $\mathbf{L}$ (for certain logics $\mathbf{L}$ ) is equivalent to $\operatorname{Alg}(\mathbf{L})$ having the amalgamation property.

In ( $\rightsquigarrow$ Craig) property $\rightsquigarrow$ is a derived connective of the logic $\mathbf{L}$ under consideration and need not be any kind of usual implication. It can be e.g. intuitionistic implication or $\square(\phi \rightarrow \psi)$ of modal logic, or $\square(\phi \rightarrow\langle$ Future $\rangle \psi)$ of temporal logic, and many others. A logic $\mathbf{L}$ has ( $\rightsquigarrow$ Craig) property if whenever $\models \phi \rightsquigarrow \psi$ there is $\chi$ using only propositional letters common in $\phi$ and $\psi$ such that $\models \phi \rightsquigarrow \chi$ and $\models \chi \rightsquigarrow \psi$. It has been proved in $[14,2]$ that the algebraic property which corresponds to ( $\rightsquigarrow$ Craig) is the superamalgamation property with respect to a relation $R_{\rightsquigarrow}$ defined from $\rightsquigarrow$. (The original version of superamalgamation has been introduced by Maksimova [16, 15] and further studied and generalized by Madarász [14]). Note that if the logic $\mathbf{L}$ in question carries a deduction theorem, then the two mentioned versions of Craig interpolation theorem can be deduced from each other (cf. [1, 2]).

There is a tradition in algebraic logic to study local versions of classical theorems of logics, e.g. one defines the notion of local explicit definition with respect to weak Beth definability property (cf. Chapter 7 in [2]) or

\footnotetext{
${ }^{1}$ The necessary definitions will be recalled in Section 2 .
} 
local Craig interpolation with respect to homogeneous first order structures [12]. Following [12] our logic $\mathbf{L}$ is said to have ( $\rightsquigarrow$ Local Craig) interpolation property if whenever $\mathfrak{M} \models \phi \rightsquigarrow \psi$ there is $\chi$ using the common atomic formulas (propositional letters) of $\phi$ and $\psi$ only, such that $\mathfrak{M} \models \phi \rightsquigarrow \chi$ and $\mathfrak{M} \models \chi \rightsquigarrow \psi$. Having propositional-like logics in mind one might be tempted to think at this point that such a property should trivially hold as either $\chi=$ True or $\chi=$ False should work. However, this is far from being the case. One reason is that $\rightsquigarrow$ need not be any kind of classical implication (as mentioned above), another reason is that our logic need not behave as a propositional logic. In Section 5 we give an easy example for two very similar propositional logics, one having ( $\rightarrow$ Local Craig) property, the other not.

In this paper we give an algebraic characterization of the mentioned local version of Craig interpolation property. In particular we show in Theorem 4.2 that ( $\rightsquigarrow$ Local Craig) interpolation (Definition 4.1 ) of a logic $\mathbf{L}$ corresponds to a strong version of the superamalgamation property (Definition 3.3).

The paper is organized as follows. In the first section we briefly recall the Andréka-Német-Sain approach to universal algebraic logic from [1, 2]. The framework is strongly related to that of Blok and Pigozzi $[5,4,6,19]$ or Font-Jansana [10, 11]; if the Reader is familiar with any of these (or similar) approaches, then we suggest skipping this section. Section 3 is devoted to the strong superamalgamation property which will be used in Theorem 4.2. In Section 4, after having defined ( $\rightsquigarrow$ Local Craig) property we give the long-promised algebraic characterization. Then in Section 5 we conclude with an easy example.

\section{Preliminary definitions}

In order to keep the paper more or less self-contained this section gives some preliminary definitions and theorems. Everything in this section can be found in a detailed form in $[1,2]$. To save space the presentation here we will be brief.

Definition 2.1 (Logic). A logic is a tuple $\mathcal{L}=\langle F, M, \mathrm{mng}, \models\rangle$ such that

- F (called the set of formulas) is a subset of finite sequences over some alphabet; 
- $M$ is a class (called the class of models);

- $\models \subseteq M \times F$ (validity relation);

- $\forall \phi, \psi \in F$ and $\mathfrak{M} \in M$ we have $\left(\operatorname{mng}_{\mathfrak{M}}(\phi)=\operatorname{mng}_{\mathfrak{M}}(\psi)\right.$ and $\left.\mathfrak{M} \models \phi\right)$ $\Longrightarrow \mathfrak{M} \models \psi$.

Definition 2.2 (Strongly nice logic). We say that $\mathcal{L}=\langle F, M, \mathrm{mng}, \models\rangle$ is strongly nice if conditions (1)-(4) below hold.

1. There is a set $C n(\mathcal{L})$ of logical connectives and a set $P$ (called atomic formulas) such that $F$ is the universe of the absolutely free algebra (word algebra) generated by $P$ in similarity type $C n$. The word algebra is denoted by $\mathcal{F}$. If we want to emphasize the role of $P$, then we write $F^{P}, \mathcal{F}^{P}$, etc.

2. The function $\operatorname{mng}_{\mathfrak{M}}$ is a homomorphism from $\mathcal{F}$ into some algebra, for every $\mathfrak{M} \in M$. (Compositionality).

3. Filter property: There are derived binary connectives $\Delta_{1}, \ldots, \Delta_{n}$ for some $n \in \omega, n>0$ and derived connectives $\varepsilon_{1}, \ldots, \varepsilon_{m}$ and $\delta_{1}, \ldots, \delta_{m}$ for some $m>0$ such that

(i) $(\forall \mathfrak{M} \in M)(\forall \phi, \psi \in F)\left(\operatorname{mng}_{\mathfrak{M}}(\phi)=\operatorname{mng}_{\mathfrak{M}}(\psi) \Leftrightarrow(\forall i \leq n) \mathfrak{M} \models\right.$ $\left.\phi \Delta_{i} \psi\right)$

(ii) $(\forall \mathfrak{M} \in M)(\forall \phi \in F)(\mathfrak{M} \models \phi \Leftrightarrow(\forall j \leq m)(\forall i \leq n) \mathfrak{M} \models$ $\left.\varepsilon_{j}(\phi) \Delta_{i} \delta_{j}(\phi)\right)$

4. $(\forall \mathfrak{M} \in M)\left(\forall h \in \operatorname{Hom}\left(\mathcal{F}, \operatorname{mng}_{\mathfrak{M}}[\mathcal{F}]\right)\right)(\exists \mathfrak{N} \in M) \operatorname{mng}_{\mathfrak{N}}=h$, where for any homomorphism $h: \mathfrak{A} \rightarrow \mathfrak{B}, h[\mathfrak{A}]$ denotes the homomorphic image of $\mathfrak{A}$ along $h$. This property is called the semantic substitution property.

As an illustration we mention that in case of classical propositional logic we can take $\varepsilon(\phi)=$ True, $\delta(\phi)=\phi$ and $\Delta=\leftrightarrow$. Then item (3) of Definition 2.2 reduces to the natural assumptions:

(i) $(\forall \mathfrak{M} \in M)(\forall \phi, \psi \in F)\left(\operatorname{mng}_{\mathfrak{M}}(\phi)=\operatorname{mng}_{\mathfrak{M}}(\psi) \Leftrightarrow \mathfrak{M} \models \phi \leftrightarrow \psi\right)$

(ii) $(\forall \mathfrak{M} \in M)(\forall \phi \in F)(\mathfrak{M} \models \phi \Leftrightarrow \mathfrak{M} \models$ True $\leftrightarrow \phi)$

In case of the modal logic $S 5$ one can also take $\phi \Delta \psi=\square(\phi \leftrightarrow \psi)$. Implicational logic, where $\rightarrow$ is the only connective is an example of a logic where $n>1$ is needed. There we can take $n=2$ and $\Delta_{1}=\rightarrow, \Delta_{2}=\leftarrow$.

Throughout the paper we will assume, for simplicity, that $n=m=1$ in Definition 2.2(3). The Readers can convince themselves that this 
assumption can easily be bypassed and this just saves us from making long formulas even longer.

Definition 2.3 (Algebraic counterpart). Let $\mathcal{L}=\langle F, M, \mathrm{mng}, \models\rangle$ be $a$ strongly nice logic.

- For $K \subseteq M$ define an equivalence relation $\sim_{K}$ on the formula algebra $\mathcal{F}$ as follows

$$
(\forall \phi, \psi \in F)\left(\phi \sim_{K} \psi \Longleftrightarrow(\forall \mathfrak{M} \in K) \operatorname{mng}_{\mathfrak{M}}(\phi)=\operatorname{mng}_{\mathfrak{M}}(\psi)\right)
$$

Since $F$ is a set $\left\{\sim_{K}: K \subseteq M\right\}$ is also a set and by Definition 2.2(2) $\sim_{K}$ is a congruence relation. Write

$$
\operatorname{Alg}_{\models}(\mathcal{L})=\mathbf{I}\left\{\mathcal{F} / \sim_{K}: K \subseteq M\right\}
$$

where $\mathbf{I}$ denotes the operation of taking isomorphic copies.

- $\operatorname{Alg}_{m}(\mathcal{L})=\left\{\operatorname{mng}_{\mathfrak{M}}[\mathcal{F}]: \mathfrak{M} \in M\right\}$.

TheOrem 2.4. For any strongly nice logic $\mathcal{L}$ we have

$$
\operatorname{Alg}_{m}(\mathcal{L}) \subseteq \operatorname{Alg}_{\models}(\mathcal{L}) \subseteq \operatorname{SPAlg}_{m}(\mathcal{L})=\operatorname{SPAlg}_{\models}(\mathcal{L})
$$

Definition 2.5 (General logic). A general logic is a class $\mathbf{L}=\left\langle\mathcal{L}^{P}: P\right.$ is a set $\rangle$, where for each set $P, \mathcal{L}^{P}=\left\langle F^{P}, M^{P}, \mathrm{mng}^{P}, \models^{P}\right\rangle$ is a logic in the sense of Definition 2.1. L is called strongly nice if conditions (1)-(4) below hold.

1. For each set $P, \mathcal{L}^{P}$ is strongly nice.

2. For any sets $P, Q, C n\left(\mathcal{L}^{P}\right)=C n\left(\mathcal{L}^{Q}\right) \stackrel{\text { def }}{=} C n(\mathbf{L})$ and the distinguished connectives $\Delta_{i}, \varepsilon_{j}, \delta_{j}$ in Definition 2.2(3) are the same.

3. If $f: P \rightarrow Q$ is a bijection, then $\mathcal{L}^{Q}$ is 'isomorphic' to $\mathcal{L}^{P}$, that is, there exists a bijection $f^{M}: M^{P} \rightarrow M^{Q}$ such that for all $\phi \in F^{P}$ and $\mathfrak{M} \in M^{P}$ we have

$$
\operatorname{mng}_{\mathfrak{M}}^{P}(\phi)=\operatorname{mng}_{f^{M}(\mathfrak{M})}^{Q}\left(f^{F}(\phi)\right),
$$

and

$$
\mathfrak{M} \models{ }^{P} \phi \Longrightarrow f^{M}(\mathfrak{M}) \models^{Q} f^{F}(\phi) .
$$

Here $f^{F}$ is the isomorphism $f^{F}: \mathcal{F}^{P} \rightarrow \mathcal{F}^{Q}$ induced by $f$. 
4. For all sets $P \subseteq Q$ we have

$$
\left\{\operatorname{mng}_{\mathfrak{M}}^{P}: \mathfrak{M} \in M^{P}\right\}=\left\{\left(\operatorname{mng}_{\mathfrak{M}}^{Q}\right) \uparrow F^{P}: \mathfrak{M} \in M^{Q}\right\}
$$

Definition 2.6 (Algebraic counterpart of a general logic). Let $\mathbf{L}=\left\langle\mathcal{L}^{P}\right.$ : $P$ is a set $\rangle$ be a strongly nice general logic. Then

$$
\begin{aligned}
& \operatorname{Alg}_{\models}(\mathbf{L})=\bigcup\left\{\operatorname{Alg}_{\models}\left(\mathcal{L}^{P}\right): P \text { is a set }\right\} \\
& \operatorname{Alg}_{m}(\mathbf{L})=\bigcup\left\{\operatorname{Alg}_{m}\left(\mathcal{L}^{P}\right): P \text { is a set }\right\}
\end{aligned}
$$

THEOREM 2.7. For a strongly nice general logic we have $\operatorname{Alg}_{=}(\mathbf{L})=$ $\mathrm{SPAlg}_{m}(\mathbf{L})$.

THEOREM 2.8. For a strongly nice general logic and a formula $\phi \in F_{\mathbf{L}}$ we have

$$
\models_{\mathbf{L}} \phi \quad \text { iff } \quad \operatorname{Alg}_{\models}(\mathbf{L}) \models \varepsilon_{j}(\phi)=\delta_{j}(\phi) \text {, for all } j<m \text {. }
$$

REMARK 2.9. For a strongly nice logic $\mathcal{L}=\langle F, M, \mathrm{mng}, \models\rangle$, a model $\mathfrak{M} \in$ $M$ and a formula $\alpha \in F$ we have the following equivalence:

$$
\begin{array}{rlr}
\mathfrak{M} \models \alpha & \Leftrightarrow \quad \mathfrak{M} \models \varepsilon(\alpha) \Delta \delta(\alpha) & \text { Definition 2.2(3)(ii) } \\
& \Leftrightarrow \operatorname{mng}_{\mathfrak{M}}(\varepsilon(\alpha))=\operatorname{mng}_{\mathfrak{M}}(\delta(\alpha)) & \text { Definition 2.2(3)(i) } \\
& \Leftrightarrow \operatorname{mng}_{\mathfrak{M}}[\mathcal{F}] \models \varepsilon(\alpha)=\delta(\alpha) &
\end{array}
$$

Finally, we will make use of the patchwork property of models:

Definition 2.10 (Patchwork property). We say that the general logic $\mathbf{L}$ has the patchwork property if

$(\forall$ sets $P, Q)\left(\forall \mathfrak{M} \in M^{P}\right)\left(\forall \mathfrak{N} \in M^{Q}\right)$

$\left(F^{P \cap Q} \neq \emptyset\right.$ and $\left.\operatorname{mng}_{\mathfrak{M}}^{P} \uparrow F^{P \cap Q}=\operatorname{mng}_{\mathfrak{N}}^{Q} \uparrow F^{P \cap Q}\right) \Longrightarrow$

$\Longrightarrow\left(\exists \mathfrak{A} \in M^{P \cup Q}\right)\left(\operatorname{mng}_{\mathfrak{A}}^{P \cup Q} \uparrow F^{P}=\operatorname{mng}_{\mathfrak{M}}^{P}\right.$ and $\left.\operatorname{mng}_{\mathfrak{A}}^{P \cup Q} \uparrow F^{Q}=\operatorname{mng}_{\mathfrak{N}}^{Q}\right)$

The patchwork property is a very natural property of logics: most of the logics discussed in the literature enjoys this property, cf. Chapter 7 in [2]. The next theorem shows that from the algebraic point of view the patchwork property is a mild assumption. 
TheOREM 2.11. For every strongly nice general logic $\mathbf{L}$ there is a strongly nice general logic $\mathbf{L}^{\prime}$ such that $\operatorname{Alg}_{\models}(\mathbf{L})=\operatorname{Alg}_{\models}\left(\mathbf{L}^{\prime}\right)$ and $\mathbf{L}^{\prime}$ has the patchwork property.

We adopted here the Andréka-Német-Sain approach to universal algebraic logic $[1,2]$. This approach originates in Tarski and others' process of algebraization of first order logic by using cylindric algebras. An other way of algebraizing logics is developed by Blok, Pigozzi $[4,5]$ and Czelakowski and is based on earlier work on logical matrices by other logicians such as Łukasiewicz and Łoś.

One of the main differences between the two approaches is the definition of what a logic is. It has been shown in Font-Jansana $[10,11]$ that the class of strongly nice general logics (Definition 2.5) is equivalent to the main class of algebraizable sentential logics in the sense of Blok-Pigozzi, moreover under some natural restrictions the classes of algebras associated with a logic by the two approaches are the same. For a comprehensive, thorough comparison of the two approaches we suggest the paper [10].

\section{The strong superamalgamation property}

Superamalgamation property goes back to Maksomiva [16, 15]. According to the original definition (cf. Definition 7.0.55 in [2]) a class $\mathrm{K}$ of partially ordered similar algebras has the superamalgamation property if for any $\mathfrak{A}_{0}, \mathfrak{A}_{1}, \mathfrak{A}_{2} \in \mathrm{K}$ and for any embeddings $i_{1}: \mathfrak{A}_{0} \rightarrow \mathfrak{A}_{1}$ and $i_{2}: \mathfrak{A}_{0} \rightarrow \mathfrak{A}_{2}$ there exists an $\mathfrak{A} \in \mathrm{K}$ and embeddings $m_{1}: \mathfrak{A}_{1} \rightarrow \mathfrak{A}, m_{2}: \mathfrak{A}_{2} \rightarrow \mathfrak{A}$ such that $m_{1} \circ i_{1}=m_{2} \circ i_{2}$ and

$\left(\forall x \in \mathfrak{A}_{j}\right)\left(\forall y \in \mathfrak{A}_{k}\right)\left(m_{j}(x) \leq m_{k}(y) \Longrightarrow\left(\exists z \in \mathfrak{A}_{0}\right)\left(x \leq i_{j}(z)\right.\right.$ and $\left.\left.i_{k}(z) \leq y\right)\right)$

where $\{j, k\}=\{1,2\}$. A slightly modified version of the superamalgamation property has been introduced in [14]. Intuitively, the main difference is that the relation therein is not necessarily an ordering but is term-definable in the following sense.

DeFINITION 3.1. Let $t$ be an algebraic similarity type and $e(x, y)$ an equation of type $t$ containing $x$ and $y$ as its only variables. Let $\mathrm{K}$ be a class of algebras of type $t$. For every $\mathfrak{A} \in \mathrm{K}$ we define a binary relation $R_{e}$ corresponding to equation e as follows:

$$
(\forall a, b \in \mathfrak{A})\left(a R_{e} b \quad \text { iff } \quad \mathfrak{A} \models e(a, b)\right)
$$


From now on we fix an algebraic similarity type $t$ and a class $\mathrm{K}$ of algebras of type $t$. We write SUPAP for superamalgamation property. Let us recall the definition of $\left(R_{e} \mathrm{SUPAP}\right)$ from [14].

Definition $3.2\left(R_{e} \mathrm{SUPAP}\right)$. Let $e(x, y)$ be an equation having $x, y$ as its only variables and $R_{e}$ the corresponding binary relation in the sense of Definition 3.1. We say that $\mathrm{K}$ has $\left(R_{e} S U P A P\right)$ if for every $\mathfrak{A}_{1}, \mathfrak{A}_{1}, \mathfrak{A}_{2} \in \mathrm{K}$ and embeddings $i_{1}: \mathfrak{A}_{0} \rightarrow \mathfrak{A}_{1}, i_{2}: \mathfrak{A}_{0} \rightarrow \mathfrak{A}_{2}$ there exists $\mathfrak{A} \in \mathrm{K}$ and embeddings $m_{1}: \mathfrak{A}_{1} \rightarrow \mathfrak{A}, m_{2}: \mathfrak{A}_{2} \rightarrow \mathfrak{A}$ such that the following diagram

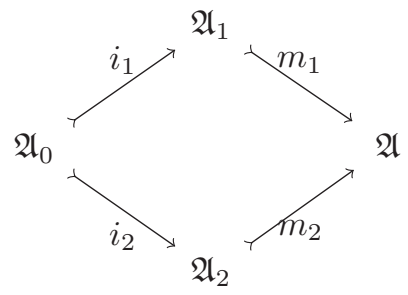

commutes and satisfies

$\left(\forall x \in \mathfrak{A}_{j}\right)\left(\forall y \in \mathfrak{A}_{k}\right)\left(m_{j}(x) R_{e} m_{k}(y) \Longrightarrow\left(\exists z \in \mathfrak{A}_{0}\right)\left(x R_{e} i_{j}(z)\right.\right.$ and $\left.\left.i_{k}(z) R_{e} y\right)\right)$ where $\{j, k\}=\{1,2\}$.

The original definition in [14] dealt with a set of equations instead of a single equation $e$. One could easily modify Definitions 3.1, 3.2 (and the forthcoming Definition 3.3) to handle the case with a set of equations. But to keep the text simple we will refrain from doing so and stick to the single equation case.

Madarász [14] also introduces the (Free $R_{e}$ SUPAP) property: $\mathrm{K}$ has the (Free $R_{e}$ SUPAP) if in Definition 3.2 we have $\mathfrak{A}_{0}=\mathfrak{F r}_{\mathrm{K}}(X \cap Y), \mathfrak{A}_{1}=$ $\mathfrak{F r}_{\mathrm{K}}(X), \mathfrak{A}_{2}=\mathfrak{F r}_{\mathrm{K}}(Y)$ and $\mathfrak{A}=\mathfrak{F r}_{\mathrm{K}}(X \cup Y)$ are $\mathrm{K}$-free algebras and the embeddings are the natural embeddings between the free algebras (for a more precise definition see Definition 4.4 in [14]).

The amalgamation property we make use of is a slight modification of Definition 3.2.

Definition 3.3 (Strong $R_{e}$ SUPAP). Let $e(x, y)$ be an equation having $x, y$ as its only variables and $R_{e}$ the corresponding binary relation in the sense of Definition 3.1. We say that $\mathrm{K}$ has (Strong $R_{e} S U P A P$ ) if for every 
$\mathfrak{A}_{0}, \mathfrak{A}_{1}, \mathfrak{A}_{2} \in \mathrm{K}$ with $\mathfrak{A}_{0} \leq \mathfrak{A}_{1}$ and $\mathfrak{A}_{0} \leq \mathfrak{A}_{2}$ there exists $\mathfrak{A} \in \mathrm{K}$ such that $\mathfrak{A}_{1} \leq \mathfrak{A}, \mathfrak{A}_{2} \leq \mathfrak{A}$ and we have

$$
\left(\forall x \in \mathfrak{A}_{j}\right)\left(\forall y \in \mathfrak{A}_{k}\right)\left(x R_{e} y \Rightarrow\left(\exists z \in \mathfrak{A}_{0}\right)\left(x R_{e} z \text { and } z R_{e} y\right)\right)
$$

where $\{j, k\}=\{1,2\}$.

The difference between $\left(R_{e}\right.$ SUPAP) and (Strong $R_{e}$ SUPAP) is that in the latter we require the embeddings $i_{1}, i_{2}, m_{1}$ and $m_{2}$ in Definition 3.2 to be the inclusion maps. Note that as $\mathfrak{A}_{i} \leq \mathfrak{A}$ it does not matter whether $x R_{e} z$ is evaluated in $\mathfrak{A}_{i}$ or in $\mathfrak{A}$, thus the definition makes sense.

\section{Local Craig interpolation}

Definition 4.1. Let $\mathbf{L}$ be a general logic and let $\rightsquigarrow$ be a derived binary connective of $\mathbf{L}$. We say that $\mathbf{L}$ has the ( $\rightsquigarrow$ Local Craig) interpolation property if for every $\phi, \psi \in F_{\mathbf{L}}$ and model $\mathfrak{M} \in M_{\mathbf{L}}$ whenever $\mathfrak{M} \models \phi \rightsquigarrow \psi$ and $(\star)$ below holds, then also $\mathfrak{M} \models \phi \rightsquigarrow \chi$ and $\mathfrak{M} \models \chi \rightsquigarrow \psi$ for some $\chi \in F_{\mathbf{L}}$ with $\operatorname{Voc}(\chi) \subseteq \operatorname{Voc}(\phi) \cap \operatorname{Voc}(\psi)$, where $\operatorname{Voc}(\alpha)$ denotes the set of atomic formulas occurring in $\alpha$, for any formula $\alpha \in F_{\mathbf{L}}$.

$$
\left\{\chi \in F_{\mathbf{L}}: \operatorname{Voc}(\chi) \subseteq \operatorname{Voc}(\phi) \cap \operatorname{Voc}(\psi)\right\} \neq \emptyset
$$

THEOREM 4.2. Let $\mathbf{L}$ be a strongly nice general logic satisfying the patchwork property. Assume $\mathbf{L}$ has a derived binary connective $\rightsquigarrow$ and let $R_{\rightsquigarrow \text { be }}$ the binary relation which corresponds to the equation $\varepsilon(x \rightsquigarrow y)=\delta(x \rightsquigarrow y)$ in the sense of Definition 3.1. Then the following statements below hold.

(i) Assume that $\mathfrak{M} \models \phi \rightsquigarrow \psi$ implies $\left\{\chi \in F_{\mathbf{L}}: \operatorname{Voc}(\chi) \subseteq \operatorname{Voc}(\phi) \cap\right.$ $\operatorname{Voc}(\psi)\} \neq \emptyset$. Then

$(\mathbf{L}$ has $(\rightsquigarrow$ Local Craig $)) \Longrightarrow\left(\operatorname{Alg}_{m}(\mathbf{L})\right.$ has $\left(\right.$ Strong $R_{\rightsquigarrow}$ SUPAP $\left.)\right)$

(ii) $(\mathbf{L}$ has $(\rightsquigarrow$ Local Craig $)) \Longleftarrow\left(\operatorname{Alg}_{m}(\mathbf{L})\right.$ has $\left(\right.$ Strong $R_{\rightsquigarrow}$ SUPAP $\left.)\right)$

(iii) Assume the similarity type of $\operatorname{Alg}(\mathbf{L})$ contains at least one constant symbol. Then

$(\mathbf{L}$ has $(\rightsquigarrow$ Local Craig $)) \Longleftrightarrow\left(\operatorname{Alg}_{m}(\mathbf{L})\right.$ has $\left(\right.$ Strong $R_{\rightsquigarrow}$ SUPAP $\left.)\right)$

Proof. (ii) Assume $\operatorname{Alg}_{m}(\mathbf{L})$ has (Strong $R_{\rightsquigarrow}$ SUPAP), let $\phi, \psi \in F_{\mathbf{L}}$ and let $\mathfrak{M} \in M_{\mathbf{L}}$ be a model such that $\mathfrak{M} \models \phi \rightsquigarrow \psi$ and $\left\{\chi \in F_{\mathbf{L}}: \operatorname{Voc}(\chi) \subseteq\right.$ 
$\operatorname{Voc}(\phi) \cap \operatorname{Voc}(\psi)\} \neq \emptyset$. We need to find $\chi \in F_{\mathbf{L}}$ with $\operatorname{Voc}(\chi) \subseteq \operatorname{Voc}(\phi) \cap$ $\operatorname{Voc}(\psi)$ such that $\mathfrak{M} \models \phi \rightsquigarrow \chi$ and $\mathfrak{M} \models \chi \rightsquigarrow \psi$ hold. Write $V=\operatorname{Voc}(\phi)$ and $W=\operatorname{Voc}(\psi)$. Take the following algebras: $\mathfrak{A}=\operatorname{mng}_{\mathfrak{M}}^{V \cup W}\left[\mathcal{F}^{V \cup W}\right]$, $\mathfrak{A}_{1}=\operatorname{mng}_{\mathfrak{M}}^{V \cup W}\left[\mathcal{F}^{V}\right], \mathfrak{A}_{2}=\operatorname{mng}_{\mathfrak{M}}^{V \cup W}\left[\mathcal{F}^{W}\right], \mathfrak{A}_{0}=\operatorname{mng}_{\mathfrak{M}}^{V \cup W}\left[\mathcal{F}^{V \cap W}\right]$.

Lemma 4.3. $\mathfrak{A}_{i} \leq \mathfrak{A}$ for $i \leq 2$ and $\mathfrak{A}_{0} \leq \mathfrak{A}_{1} \cap \mathfrak{A}_{2}$. For all $i \leq 2$ we have $\mathfrak{A}_{i}, \mathfrak{A} \in \operatorname{Alg}_{m}(\mathbf{L})$.

Proof. That $\mathfrak{A}_{0} \leq \mathfrak{A}_{1}$ and $\mathfrak{A}_{0} \leq \mathfrak{A}_{2}$ follows from the very fact that $\mathcal{F}^{V \cap W} \leq \mathcal{F}^{V} \cap \mathcal{F}^{W}$ and that $\operatorname{mng}_{\mathfrak{M}}^{V \cup W}$ is a homomorphism. Thus we need to verify $\mathfrak{A}_{0}, \mathfrak{A}_{1}, \mathfrak{A}_{2} \in \operatorname{Alg}_{m}(\mathbf{L})$. We prove more: If $\mathbf{L}$ is strongly nice, then $\mathrm{Alg}_{m}(\mathbf{L})$ is closed under taking subalgebras.

To this end let $\mathfrak{A} \in \operatorname{Alg}_{m}(\mathbf{L})$ and $\mathfrak{B} \leq \mathfrak{A}$. We prove $\mathfrak{B} \in \operatorname{Alg}_{m}(\mathbf{L})$. Take any surjection $g: A \rightarrow B$ and let $\bar{g}: \mathcal{F}^{A} \rightarrow \mathfrak{B}$ be the unique homomorphism that extends $g$. By the substitution property (Definition 2.2(4)) there is a model $\mathfrak{M} \in M^{A}$ with $\bar{g}=\operatorname{mng}_{\mathfrak{M}}^{A}$ and therefore $\mathfrak{B}=\operatorname{mng}_{\mathfrak{M}}^{A}\left[\mathcal{F}^{A}\right] \in \operatorname{Alg}_{m}(\mathbf{L})$.

Let now $x=\operatorname{mng}_{\mathfrak{M}}^{V \cup W}(\phi)$ and $y=\operatorname{mng}_{\mathfrak{M}}^{V \cup W}(\psi)$. By the filter property (Definition 2.2(3)) and Remark 2.9, M $=\phi \rightsquigarrow \psi$ is equivalent to $\mathfrak{A} \models x R_{\rightsquigarrow} y$. By (Strong $R_{\rightsquigarrow}$ SUPAP) of $\operatorname{Alg}_{m}(\mathbf{L})$ there is $z \in \mathfrak{A}_{0}$ such that $x R_{\rightsquigarrow} z$ and $z R_{\rightsquigarrow} y$. As $z \in \mathfrak{A}_{0}$ there must exists some $\chi \in F^{V \cap W}$ such that $z=\operatorname{mng}_{\mathfrak{M}}^{V U W}(\chi)$. Then $x R_{\rightsquigarrow z} z$ implies $\mathfrak{M} \models \phi \rightsquigarrow \chi$ and $z R_{\rightsquigarrow} y$ implies $\mathfrak{M} \models \chi \rightsquigarrow \psi$.

(i) Assume that $\mathfrak{M} \models \phi \rightsquigarrow \psi$ implies $\left\{\chi \in F_{\mathbf{L}}: \operatorname{Voc}(\chi) \subseteq \operatorname{Voc}(\phi) \cap\right.$ $\operatorname{Voc}(\psi)\} \neq \emptyset$ and that $\mathbf{L}$ has $(\rightsquigarrow$ Local Craig). In order to show that $\operatorname{Alg}(\mathbf{L})$ has (Strong $R_{\rightsquigarrow}$ SUPAP) pick algebras $\mathfrak{A}_{0}, \mathfrak{A}_{1}, \mathfrak{A}_{2} \in \operatorname{Alg}{ }_{m}(\mathbf{L})$ such that $\mathfrak{A}_{0} \leq \mathfrak{A}_{1} \cap \mathfrak{A}_{2}$.

LEMMA 4.4. If $\mathbf{L}$ is a strongly nice general logic that has the patchwork property, then for every $\mathfrak{A}_{0}, \mathfrak{A}_{1}, \mathfrak{A}_{2} \in \operatorname{Alg}{ }_{m}(\mathbf{L})$ with $\mathfrak{A}_{0} \leq \mathfrak{A}_{1} \cap \mathfrak{A}_{2}$ there is $\mathfrak{A} \in \operatorname{Alg}_{m}(\mathbf{L})$ such that $\mathfrak{A}_{1} \leq \mathfrak{A}$ and $\mathfrak{A}_{2} \leq \mathfrak{A}$.

Proof. Suppose $\mathfrak{A}_{0}, \mathfrak{A}_{1}, \mathfrak{A}_{2} \in \operatorname{Alg} g_{m}(\mathbf{L})$ are such that $\mathfrak{A}_{0} \leq \mathfrak{A}_{1}$ and $\mathfrak{A}_{0} \leq \mathfrak{A}_{2}$. Let $f: A_{1} \rightarrow A_{1}$ and $g: A_{2} \rightarrow A_{2}$ be the identity mappings. Then $f$ and $g$ extend to homomorphisms $\bar{f}: \mathcal{F}^{A_{1}} \rightarrow \mathfrak{A}_{1}$ and $\bar{g}: \mathcal{F}^{A_{2}} \rightarrow \mathfrak{A}_{2}$. By the substitution property of $\mathbf{L}$ (Definition 2.2(4)) there are models $\mathfrak{M} \in M^{A_{1}}$ and $\mathfrak{N} \in M^{A_{2}}$ so that $\bar{f}=\operatorname{mng}_{\mathfrak{M}}^{A_{1}}$ and $\bar{g}=\operatorname{mng}_{\mathfrak{N}}^{A_{2}}$. By the 
patchwork property (Definition 2.10) for some model $\mathfrak{D} \in M^{A_{1} \cup A_{2}}$ we have $\operatorname{mng}_{\mathfrak{D}}^{A_{1} \cup A_{2}} \uparrow F^{A_{1}}=\operatorname{mng}_{\mathfrak{M}}^{A_{1}}$ and $\operatorname{mng}_{\mathfrak{D}}^{A_{1} \cup A_{2}} \uparrow F^{A_{2}}=\operatorname{mng}_{\mathfrak{N}}^{A_{2}}$. It follows that $\mathfrak{A}_{1}=\operatorname{mng}_{\mathfrak{M}}^{A_{1}}\left[\mathcal{F}^{A_{1}}\right] \leq \operatorname{mng}_{\mathfrak{D}}^{A_{1} \cup A_{2}}\left[\mathcal{F}^{A_{1} \cup A_{2}}\right]$ and $\mathfrak{A}_{2}=\operatorname{mng}_{\mathfrak{N}}^{A_{2}}\left[\mathcal{F}^{A_{2}}\right] \leq$ $\operatorname{mng}_{\mathfrak{D}}^{A_{1} \cup A_{2}}\left[\mathcal{F}^{A_{1} \cup A_{2}}\right]$.

Let $\mathfrak{A}$ be as in Lemma 4.4. As $\mathfrak{A} \in \operatorname{Alg}_{m}(\mathbf{L})$ it is the image of the meaning function with respect to some model $\mathfrak{M}$, i.e. $\mathfrak{A}=\operatorname{mng}_{\mathfrak{M}}^{A}\left[\mathcal{F}^{A}\right]$. Then $\mathfrak{A}_{1}=\operatorname{mng}_{\mathfrak{M}}^{A}\left[\mathcal{F}^{A_{1}}\right], \mathfrak{A}_{2}=\operatorname{mng}_{\mathfrak{M}}^{A}\left[\mathcal{F}^{A_{2}}\right]$ and $\mathfrak{A}_{0}=\operatorname{mng}_{\mathfrak{M}}^{A}\left[\mathcal{F}^{A_{0}}\right]$. Suppose now that for some $x \in \mathfrak{A}_{1}$ and $y \in \mathfrak{A}_{2}$ we have $x R_{\rightsquigarrow} y$. By definition there are formulas $\phi \in F^{A_{1}}$ and $\psi \in F^{A_{2}}$ such that $\operatorname{mng}_{\mathfrak{M}}^{A}(\phi)=x$ and $\operatorname{mng}_{\mathfrak{M}}^{A}(\psi)=y$. By Remark 2.9, $\mathfrak{A} \models x R_{\rightsquigarrow} y$ is equivalent to $\mathfrak{M} \models \phi \rightsquigarrow \psi$. By assumption $\left\{\chi \in F_{\mathbf{L}}: \operatorname{Voc}(\chi) \subseteq \operatorname{Voc}(\phi) \cap \operatorname{Voc}(\psi)\right\} \neq \emptyset$. Using ( $\rightsquigarrow$ Local Craig) property of $\mathbf{L}$ there is a formula $\chi \in F^{A_{1} \cap A_{2}}$ such that $\mathfrak{M} \models \phi \rightsquigarrow \chi$ and $\mathfrak{M} \models \chi \rightsquigarrow \psi$. Clearly $z=\operatorname{mng}_{\mathfrak{M}}^{A}(\chi) \in \mathfrak{A}_{0}$ and it follows that $x R_{\rightsquigarrow} z$ and $z R_{\rightsquigarrow} y$.

(iii) If the similarity type of $\operatorname{Alg}(\mathbf{L})$ contains at least one constant symbol then for every $\phi, \psi \in F_{\mathbf{L}}$ the set $\left\{\chi \in F_{\mathbf{L}}: \operatorname{Voc}(\chi) \subseteq \operatorname{Voc}(\phi) \cap \operatorname{Voc}(\psi)\right\}$ is nonempty. Thus the statement follows from (i) and (ii).

\section{Example: Sentential logic(s)}

First we recall from e.g. [1,2] two usual ways of defining sentential logic. In particular we define the logics $\mathbf{L}_{S}$ and $\mathbf{L}_{S}^{\prime}$. In each case the set of logical connectives is $C n=\{\wedge, \neg\}$ and for a set $P$ of atomic formulas the set of formulas $F^{P}$ is defined as the universe of the absolutely free algebra generated by $P$ in similarity type $C n$. The difference is in the definition of the models, mng and $\models$.

Classical sentential logic $\mathbf{L}_{S}$. Fix a set $P$. In classical sentential logic a model $\mathfrak{M} \in M^{P}$ is a function assigning 1 (true) or 0 (false) to each atomic proposition $p \in P$. Any such mapping extends in a usual way to a unique mapping $F^{P} \rightarrow 2$ (which we also denote by $\mathfrak{M}$ ). We define $\operatorname{mng}_{\mathfrak{M}}^{P}$ and $\models^{P}$ for all formulas $\varphi \in F^{P}$ as follows.

$$
\begin{aligned}
& \operatorname{mng}_{\mathfrak{M}}^{P}(\varphi)=\mathfrak{M}(\varphi) \\
& \mathfrak{M} \models^{P} \varphi \quad \text { if and only if } \quad \mathfrak{M}(\varphi)=1
\end{aligned}
$$


Finally, $\mathbf{L}_{S}=\left\langle\left\langle F^{P}, M^{P}, \operatorname{mng}^{P}, \models^{P}\right\rangle: \quad P\right.$ is a set $\rangle$. The two classes of algebras corresponding to $\mathbf{L}_{S}$ are (see Chapter 7 of [1])

$$
\begin{aligned}
& \operatorname{Alg}_{\models}\left(\mathbf{L}_{S}\right)=\mathrm{BA} \\
& \operatorname{Alg}_{m}\left(\mathbf{L}_{S}\right)=\{\mathbf{2}\}
\end{aligned}
$$

where BA is the class of all Boolean algebras and $\mathbf{2}$ is the 2-element Boolean algebra.

Sentential logic as modal logic $\mathbf{L}_{S}^{\prime}$. The set of connectives and for any set $P$ the set of formulas are like in the previous case. The class of models for the set $P$ of atomic formulas is

$$
M_{S}^{\prime}=\{\langle W, v\rangle: W \neq \emptyset, v: P \rightarrow \mathcal{P}(W)\} .
$$

For a model $\mathfrak{M}=\langle W, v\rangle, w \in W$ and a formula $\varphi$ one can define $\mathfrak{M}, w \Vdash \varphi$ in the usual modal logic way (cf. p.97 of [1]). We let the meaning function $\operatorname{mng}_{\mathfrak{M}}: \mathcal{F}^{P} \rightarrow\langle\mathcal{P}(W), \cap, \backslash\rangle$ to be the homomorphic extension such that

$$
\begin{array}{cll}
\mathfrak{M}, w \Vdash \varphi & \text { IFF } & w \in \operatorname{mng}_{\mathfrak{M}}(\varphi), \text { and } \\
\mathfrak{M} \models \varphi & \text { IFF } & W=\operatorname{mng}_{\mathfrak{M}}(\varphi) .
\end{array}
$$

This defines the general logic $\mathbf{L}_{S}^{\prime}$. The two classes of algebras corresponding to $\mathbf{L}_{S}^{\prime}$ are (see Chapter 7 of [1])

$$
\begin{aligned}
& \operatorname{Alg}_{\models}\left(\mathbf{L}_{S}^{\prime}\right)=\mathrm{BA} \\
& \operatorname{Alg}_{m}\left(\mathbf{L}_{S}^{\prime}\right)=\operatorname{setBA}
\end{aligned}
$$

where setBA $=\mathbf{S}\{\langle\mathcal{P}(W), \cap, \backslash\rangle: W$ is a nonempty set $\}$ is the class of all non-trivial set Boolean algebras.

Both $\mathbf{L}_{S}$ and $\mathbf{L}_{S}^{\prime}$ has a derived connective $\rightarrow$ defined in the usual way that corresponds to the usual Boolean ordering $\leq$. E.g. Theorem 4.2(ii) reads as

$$
\left(\operatorname{Alg}_{m}\left(\mathbf{L}_{S}\right) \text { has }(\text { Strong } \leq \text { SUPAP })\right) \Longrightarrow\left(\mathbf{L}_{S} \text { has }(\rightarrow \text { Local Craig })\right)
$$

It is straightforward to check that $\mathbf{L}_{S}$ has ( $\rightarrow$ Local Craig) interpolation: on the one hand $\operatorname{Alg}_{m}\left(\mathbf{L}_{S}\right)=\{\mathbf{2}\}$ obviously has (Strong $\leq$ SUPAP) and thus Theorem 4.2(ii) applies, on the other hand if $\mathfrak{M} \models \phi \rightarrow \psi$, then either $\chi=x \wedge \neg x$ or its negation is a suitable interpolant formula. 
But $\operatorname{Alg}_{m}\left(\mathbf{L}_{S}^{\prime}\right)$ does not have (Strong $\leq$ SUPAP): take $\mathfrak{A}_{1}=\mathfrak{A}_{2}=$ $\langle\mathcal{P}(\{a, b, c\}), \cap, \backslash\rangle$ and let $\mathfrak{A}_{0}$ be the subalgebra of $\mathfrak{A}_{1}$ generated by the element $a$. Then we have $b \leq c^{\perp}$ but neither $a$ nor $a^{\perp}$ is in between $b$ and $c^{\perp}$. A very easy argument shows that $\mathbf{L}_{S}^{\prime}$ does not have $(\rightarrow$ Local Craig) interpolation either.

ACKNowledgement. I am grateful for the two anonymous referees for all their suggestions, the paper has benefited from their careful reading. I wish to acknowledge the Premium Postdoctoral Grant of the Hungarian Academy of Sciences.

\section{References}

[1] H. Andréka, I. Németi, I. Sain, Algebraic Logic, [in:] D. M. Gabbay and F. Guenthner (eds.), Handbook of Philosophical Logic Vol. II, Second edition, Kluwer Academic Publishers, 2001, pp. 133-247.

[2] H. Andréka, I. Németi, I. Sain, Universal Algebraic Logic, Studies in Logic, Springer, due to 2017.

[3] H. Andréka, Á. Kurucz, I. Németi, I. Sain, Applying algebraic logic; A general methodology, Lecture Notes of the Summer School "Algebraic Logic and the Methodology of Applying it", Budapest 1994.

[4] W. J. Blok, D. Pigozzi, Algebraizable logics, Memoirs of the American Mathematical Society, Vol. 77, No. 396, pp. vi+78, 1989.

[5] W. J. Blok, D. Pigozzi, Abstract Algebraic Logic, Lecture Notes of the Summer School "Algebraic Logic and the Methodology of Applying it", Budapest 1994.

[6] W. J. Blok, D. Pigozzi, Local Deduction Theorems in Algebraic Logic, [in:] J. D. Monk, H. Andréka and I. Németi (eds.), Algebraic Logic (Proc. Conf. Budapest 1988), Vol. 54 of Colloq. Math. Soc. János Bolyai, NorthHolland, Amsterdam, 1991, pp. 75-109.

[7] J. Czelakowski, Logical matrices and the amalgamation property, Studia Logica XLI(4) (1982), pp. 329-342.

[8] J. Czelakowski, D. Pigozzi, Amalgamation and Interpolation in abstract algebraic logic, [in:] Models, Algebras, and Proofs, selected papers of the X Latin American symposium on mathematical logic held in Bogotá, Xavier Caicedo and Carlos H. Montenegro (eds.), Lecture Notes in Pure and Applied Mathematics, Vol. 203, Marcel Dekker, Inc., New York, 1999. 
[9] J. Czelakowski, D. Pigozzi. Fregean logics, Annals of Pure and Applied Logic 127(1/3) (2004), pp. 17-76.

[10] J. M. Font, R. Jansana, A comparison of two approaches to the algebraization of logics, Lecture Notes of the Summer School "Algebraic Logic and the Methodology of Applying it", Budapest 1994.

[11] J. M. Font, R. Jansana, On the Sentential Logics Associated with strongly nice and Semi-nice General Logics, Bulletin of the IGPL, Vol. 2, No. 1 (1994), pp. 55-76.

[12] Z. Gyenis, Interpolation property and homogeneous structures, Logic Journal of IGPL 22(4) (2014), pp. 597-607.

[13] L. Henkin, J. D. Monk, A. Tarski, Cylindric Algebras Parts I, II, North Holland, Amsterdam, 1971.

[14] J. Madarász, Interpolation and Amalgamation, Pushing the Limits. Part I and Part II, Studia Logica, Vol. 61 and 62(3 and 1), (1998 and 1999), pp. 311-345 and pp. 1-19.

[15] L. L. Maksimova, Amalgamation and interpolation in normal modal logics, Studia Logica L(3/4) (1991), pp. 457-471.

[16] L. L. Maksimova, Interpolation theorems in modal logics and amalgamable varieties of topoboolean algebras, (in Russian), Algebra i logika 18, 5 (1979), pp. 556-586.

[17] D. Nyíri, Robinson's property and amalgamations of higher arities, Mathematical Logic Quarterly, Vol. 62, Issue 4-5 (2016), pp. 427-433.

[18] D. Pigozzi, Amalgamation, Congruence Extension and Interpolation Properties in Algebras, Algebra Universalis 1(3) (1972), pp. 269-349.

[19] D. Pigozzi, Fregean algebraic logic, [in:] J. D. Monk, H. Andréka and I. Németi (eds.), Algebraic Logic (Proc. Conf. Budapest 1988), Vol. 54 of Colloq. Math. Soc. János Bolyai, North-Holland, Amsterdam, 1991, pp. 475-502.

[20] I. Sain, Beth's and Craig's properties via epimorphisms and amalgamation in algebraic logic, Algebraic Logic and Universal Algebra in Computer Science, Bergman, Maddux and Pigozzi (eds.), Lecture Notes in Computer Science, Vol. 425, Springer-Verlag, Berlin, 1990, pp. 209-226.

[21] G. Sági, S. Shelah, On Weak and Strong Interpolation in Algebraic Logics, Journal of Symbolic Logic, Vol. 71, No. 1, (2006), pp. 104-118.

Department of Logic, Jagiellonian University, Kraków, and

Department of Logic, Eötvös University, Budapest

e-mail: gyz@renyi.hu 